\title{
Electron Scattering Through a Quantum Dot
}

\author{
Leonardo Kleber Castelano ${ }^{1}$, Guo-Qiang Hai $^{2}$ and Mu-Tao Lee ${ }^{3}$ \\ ${ }^{1}$ Departamento de Física, Universidade Federal de São Carlos \\ ${ }^{2}$ Instituto de Física de São Carlos, Universidade de São Paulo \\ ${ }^{3}$ Departamento de Química, Universidade Federal de São Carlos \\ Brazil
}

\section{Introduction}

Electron scattering and transport through quantum dots (QDs) in a semiconductor nanostructure have been intensively studied (Engel \& Loss, 2002; Fransson et al., 2003; Konig \& Martinek, 2003; Koppens et al., 2006; Qu \& Vasilopoulos, 2006; Zhang et al., 2002). The spin-dependent transport properties are of particular interest for its possible applications, e.g., the QD spin valves (Konig \& Martinek, 2003), the quantum logic gates using coupled QDs, as well as the spin-dependent transport in single-electron devices (Seneor et al., 2007), etc.. In such systems, the electron-electron exchange potential and the electron spin states have been utilized and manipulated (Burkard et al., 2000; Gundogdu et al., 2004; Sarma et al., 2001; Wolf et al., 2001). A thorough quantitative understanding of spin-dependent transport properties due to electron-electron interaction is therefore important for a successful construction of these devices. Theoretically the transport through QDs has been studied by different approaches such as transfer matrix, nonequilibrium Green's functions, random matrix theory, as well as those methods built on the Lippmann-Schwinger (L-S) equation (Castelano et al., 2007a;b).

In this chapter, we develop a theoretical method to study electron scattering through a quantum dot (QD) of N-electrons embedded in a semiconductor nanostructure. We construct the scattering equations including electron-electron interaction to represent the process of a free electron scattered by the QD confined in a two-dimensional (2D) or in a quasi-one-dimensional (Q1D) semiconductor system. The generalized multichannel Lippmann-Schwinger equations(Bransden \& McDowell, 1977; Joachain, 1975) are solved for these systems by using the method of continued fractions (MCF). As an example, we apply this method to a one-electron QD case and obtain scattering cross-sections in 2D and conductances in Q1D systems resulting from both the singlet- and triplet-coupled continuum states of two electrons (incident and QD electron) during the electron transport.

This chapter is organized as follows. In Sec. 2 we present our general theoretical approach and numerical method. In Sec. 3, we describe the electron scattering through a quantum dot in a 2D system. The scattering for a quantum dot confined in a Q1D system is presented in Sec. 4. In Sections 5 and 6, we show our numerical results for the scattering through a one-electron QD within both the one-channel and the multichannel models. We conclude in Sec. 7. 


\section{Theoretical approach}

\subsection{The system: Incident electron + quantum dot}

The system under investigation consists of an incident free electron and a quantum dot of $\mathrm{N}$ electrons as shown schematically in Fig. 1. The incident electron is scattered by both the QD potential and by the confined electrons inside the QD. The Schrödinger equation of the system is given by

$$
\left(H-\mathcal{E}_{i}\right) \Psi_{i}\left(\tau ; \mathbf{r}_{N+1}, \sigma_{N+1}\right)=0,
$$

where $\tau$ represents collectively the spatial and spin coordinates of the $\mathrm{N}$ electrons localized in the QD and $\mathbf{r}_{N+1}=\left(x_{N+1}, y_{N+1}\right)$ and $\sigma_{N+1}$ denote the spatial and spin coordinates of the incident electron. The total energy of the system is $\mathcal{E}_{i}$, where the subscript $i$ represents a set of quantum numbers required to uniquely specify the initial quantum state of the system. Explicitly, the total Hamiltonian of the system can be written as

$$
H=H_{0}\left(\mathbf{r}_{N+1}\right)+H_{\mathrm{QD}}(\tau)+V_{\text {int }}\left(\mathbf{r}_{1}, \mathbf{r}_{2}, \ldots, \mathbf{r}_{N}, \mathbf{r}_{N+1}\right),
$$

where $H_{0}\left(\mathbf{r}_{N+1}\right)=-\hbar^{2} \nabla_{N+1}^{2} / 2 m^{*}+V_{\mathrm{QD}}\left(\mathbf{r}_{N+1}\right), H_{\mathrm{QD}}(\tau)$ is the Hamiltonian of the QD of $\mathrm{N}$ electrons, and $V_{\text {int }}$ is the interaction potential between the incident electron at $\mathbf{r}_{N+1}$ and the $\mathrm{N}$ electrons in the QD

$$
V_{\text {int }}\left(\mathbf{r}_{1}, \mathbf{r}_{2}, \ldots, \mathbf{r}_{N}, \mathbf{r}_{N+1}\right)=\frac{e^{2}}{\epsilon_{0}^{*}} \sum_{i=1}^{N} \frac{1}{\left|\mathbf{r}_{N+1}-\mathbf{r}_{i}\right|},
$$

where $\epsilon_{0}^{*}$ is the dielectric constant of the semiconductor material and $m^{*}$ is the electron effective mass. The Hamiltonian for an unperturbed QD is given by

$$
H_{\mathrm{QD}}(\tau)=\sum_{i=1}^{N}\left(-\frac{\hbar^{2}}{2 m^{*}} \nabla_{i}^{2}+V_{\mathrm{QD}}\left(\mathbf{r}_{\mathbf{i}}\right)\right)+\frac{e^{2}}{\epsilon_{0}^{*}} \sum_{i \neq j}^{N} \frac{1}{\left|\mathbf{r}_{i}-\mathbf{r}_{j}\right|},
$$

where the first term in the rhs of Eq. (4) describes $\mathrm{N}$ independent electrons in the QD of confinement potential $V_{\mathrm{QD}}(\mathbf{r})$ and the second term gives the Coulomb interactions among these electrons. The eigenenergy and eigenfunction of this $\mathrm{N}$-electron QD are denoted by $\varepsilon_{n}$ and $\Phi^{n}$, respectively. They are determined by the following Schrödinger equation

$$
H_{\mathrm{QD}}(\tau) \Phi^{n}=\varepsilon_{n} \Phi^{n}
$$

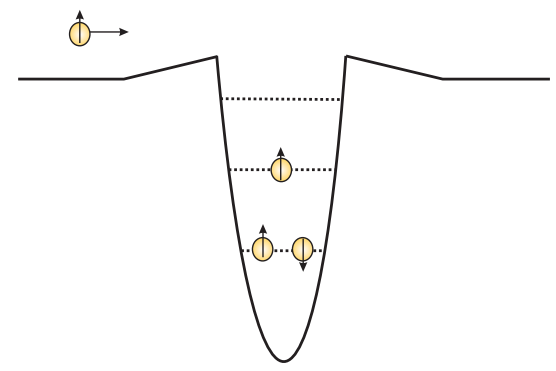

Fig. 1. Representation of the incident electron and the target, which in this case is a quantum dot containing 3 electrons. 
with $n=0,1,2,3 \ldots$. The ground state of the QD is labeled by $n=0$ and the excited states by $n \geq 1$. The eigenstates of the QD can be obtained using, e.g., the restricted or unrestricted Hartree-Fock (HF) methods (Szabo \& Ostlund, 1982).

\subsection{Scattering equations including electron-electron interaction}

In order to extract scattering properties of the system (QD + incident electron), we can write the total wave-function $\Psi_{i}$ of the system as a superposition of the QD wave-function $\Phi^{n}$ and the incident electron wave-function,

$$
\left|\Psi_{i}\right\rangle=\sum_{n=0}^{\infty}\left|\mathcal{A}\left(\Phi^{n} \psi_{n i}\right)\right\rangle
$$

where $\psi_{n i}$ describes the wave-functions of the incident (scattered) electron in the continuum states corresponding to a quantum transition from an initial state $i$ to a final state $n$. The operator $\mathcal{A}$ warrants the antisymmetrization property between the QD electrons and the incident electron, defined by,

$$
\mathcal{A}=\frac{1}{\sqrt{N+1}} \sum_{p=1}^{N+1}(-1)^{N+1-p} \mathcal{P}_{N+1, p}
$$

where $\mathcal{P}_{N+1, p}$ is the permutation operator which exchanges the electrons at $\mathbf{r}_{N+1}$ and $\mathbf{r}_{p}$. From Eqs. (1), (2) and (6), we obtain

$$
\sum_{n=0}^{\infty}\left(-\frac{\hbar^{2}}{2 m^{*}} \nabla_{N+1}^{2}+V_{\mathrm{QD}}+H_{\mathrm{QD}}+V_{\mathrm{int}}\right)\left|\mathcal{A}\left(\Phi^{n} \psi_{n i}\right)\right\rangle=\mathcal{E}_{i} \sum_{n=0}^{\infty}\left|\mathcal{A}\left(\Phi^{n} \psi_{n i}\right)\right\rangle .
$$

The total energy of the system $\mathcal{E}_{i}$ is composed of two parts. The first part is the kinetic energy of the incident (scattering) electron and the second is the energy of the N-electron QD in a particular configuration, i.e., $\mathcal{E}_{i}=\frac{\hbar^{2} k_{i}^{2}}{2 m^{*}}+\varepsilon_{i}=\frac{\hbar^{2} k_{n}^{2}}{2 m^{*}}+\varepsilon_{n}$, for different eigenstates of the QD $(i, n=0,1,2, \ldots)$ or different scattering channels. These different channels appear because the incident electron can probably be scattered inelastically, leaving the QD in a different state from its initial. A projection of Eq. (8) onto a particular QD state $\left|\Phi^{m}\right\rangle$ leads to the following scattering equation for the incident electron,

$$
\frac{\hbar^{2}}{2 m^{*}}\left(\nabla^{2}+k_{m}^{2}\right) \psi_{m i}(\mathbf{r})=\sum_{n=0}^{\infty} V_{m n}(\mathbf{r}) \psi_{n i}(\mathbf{r})
$$

for $i, m=0,1,2, \ldots$, where $\mathbf{r}=\mathbf{r}_{N+1}$ and $V_{m n}=V_{m n}^{\text {st }}+V_{m n}^{\text {ex }}$ with $V_{m n}^{\text {st }}$ the static potential and $V_{m n}^{\mathrm{ex}}$ the exchange potential due the nonlocal interaction, giving by

$$
V_{m n}^{\mathrm{st}}(\mathbf{r})=V_{\mathrm{QD}}(\mathbf{r}) \delta_{m n}+\frac{e^{2}}{\epsilon_{0}^{*}} \sum_{j=1}^{N}\left\langle\Phi^{m}\left|\frac{e^{-\lambda\left|\mathbf{r}-\mathbf{r}_{\mathbf{j}}\right|}}{\left|\mathbf{r}-\mathbf{r}_{\mathbf{j}}\right|}\right| \Phi^{n}\right\rangle,
$$


and

$$
V_{m n}^{\mathrm{ex}}(\mathbf{r}) \psi_{n i}(\mathbf{r})=\left(H_{0}(\mathbf{r})-\frac{\hbar^{2} k_{m}^{2}}{2 m^{*}}\right)\left\langle\Phi^{m} \mid \mathcal{A}^{\prime}\left(\Phi^{n} \psi_{n i}\right)\right\rangle+\frac{e^{2}}{\epsilon_{0}^{*}} \sum_{j=1}^{N}\left\langle\Phi^{m}\left|\frac{1}{\left|\mathbf{r}-\mathbf{r}_{\mathbf{j}}\right|}\right| \mathcal{A}^{\prime}\left(\Phi^{n} \psi_{n i}\right)\right\rangle,
$$

respectively, where $\mathcal{A}^{\prime}=\sum_{p=1}^{N}(-1)^{N+1-p} \mathcal{P}_{N+1, p}$. In Eq. (10) we have introduced a screening $e^{-\lambda\left|\mathbf{r}-\mathbf{r}^{\prime}\right|}$ on the direct Coulomb potential for two reasons: (i) the ionized impurities in the semiconductor nanostructure and/or the external electrodes screen the direct Coulomb potential and (ii) at $|\mathbf{r}| \rightarrow \infty$ limit the scattering potential should decay faster than $1 /|\mathbf{r}|$. The screening length is given by $\lambda^{-1}$. Notice that we do not consider the screening on the exchange potential because this potential is non-zero inside the QD only. Inclusion of the screening on the exchange potential in Eq. (11) is possible but it will not affect much our results and considerably complicates the numerical calculation. The scattering equation is a system of coupled integro-differential equations. The corresponding generalized L-S equation for such a multichannel scattering problem is given by

$$
\psi_{m i}(\mathbf{r})=\varphi_{i}(\mathbf{r}) \delta_{m i}+\sum_{n=0}^{\infty} \int d \mathbf{r}^{\prime} G^{(0)}\left(\mathbf{k}_{m}, \mathbf{r}, \mathbf{r}^{\prime}\right) V_{m n}\left(\mathbf{r}^{\prime}\right) \psi_{n i}\left(\mathbf{r}^{\prime}\right), \text { for } i, m=0,1,2 \ldots
$$

with an incident plane wave $\varphi_{i}(\mathbf{r})=e^{i \mathbf{k}_{i} \cdot \mathbf{r}}=e^{i k_{i} x}$ in the $x$-direction.

\subsection{Method of continued fractions}

The method of continued fractions (MCF) (Horacek \& Sasakawa, 1984) is an iterative method to solve the L-S equation. To apply this method for a multi-channel scattering we have firstly to rewrite Eq. (12) in a matrix form:

$$
\widetilde{\Psi}=\widetilde{\varphi}+\widetilde{G}^{(0)} \widetilde{V} \widetilde{\Psi}
$$

In the first step to start the MCF, we use the scattering potential $\widetilde{V}=V^{(0)}$ and the free electron wave-function $\widetilde{\varphi}=\left|\varphi^{(0)}\right\rangle$ in Eq. (13). Afterwards, we define the $\mathrm{n}$ th-order weakened potential as

$$
V^{(n)}=V^{(n-1)}-\frac{V^{(n-1)}\left|\varphi^{(n-1)}\right\rangle\left\langle\varphi^{(n-1)}\right| V^{(n-1)}}{\left\langle\varphi^{(n-1)}\left|V^{(n-1)}\right| \varphi^{(n-1)}\right\rangle},
$$

where

$$
\left|\varphi^{(n)}\right\rangle=\widetilde{G}^{(0)} V^{(n-1)}\left|\varphi^{(n-1)}\right\rangle .
$$

The $\mathrm{n}$ th-order correction of the $\mathrm{T}$ matrix can be obtained through

$$
\begin{array}{r}
T^{(n)}=\left\langle\varphi^{(n-1)}\left|V^{(n-1)}\right| \varphi^{(n)}\right\rangle+\left\langle\varphi^{(n)}\left|V^{(n)}\right| \varphi^{(n)}\right\rangle \\
\times\left[\left\langle\varphi^{(n)}\left|V^{(n)}\right| \varphi^{(n)}\right\rangle-T^{(n+1)}\right]^{-1}\left\langle\varphi^{(n)}\left|V^{(n)}\right| \varphi^{(n)}\right\rangle .
\end{array}
$$

Hence, we can stop the iteration when the potential $V^{(N)}$ becomes weaker enough. In the numerical calculation, we start with $T^{(N+1)}=0$ and evaluate $T^{(N)}, T^{(N-1)}, \ldots$, and $T^{(1)}$. 
Therefore the $\mathbf{T}$ matrix is given by

$$
\mathbf{T}=\left\langle\varphi^{(0)}\left|V^{(0)}\right| \varphi^{(0)}\right\rangle+T^{(1)} \frac{\left\langle\varphi^{(0)}\left|V^{(0)}\right| \varphi^{(0)}\right\rangle}{\left\langle\varphi^{(0)}\left|V^{(0)}\right| \varphi^{(0)}\right\rangle-T^{(1)}} .
$$

\section{Quantum dot embedded in a two-dimensional system}

The Green's function $G^{(0)}\left(\mathbf{k}, \mathbf{r}, \mathbf{r}^{\prime}\right)$ in $2 \mathrm{D}$ is given by

$$
G^{(0)}\left(\mathbf{k}, \mathbf{r}, \mathbf{r}^{\prime}\right)=-\frac{2 m^{*}}{\hbar^{2}}(i / 4) H_{0}^{(1)}\left(k\left|\mathbf{r}-\mathbf{r}^{\prime}\right|\right),
$$

where $H_{0}^{(1)}$ is the usual zero order Hankel's function (Morse \& Feshbach, 1953).

At $|\mathbf{r}| \rightarrow \infty$ limit, the asymptotic form of Eq. (12) for the scattered wave-function in a 2D system is given by

$$
\psi_{m i}(\mathbf{r}) \underset{|\mathbf{r}| \rightarrow \infty}{\longrightarrow} e^{i k_{i} x} \delta_{m i}+\frac{2 m^{*}}{\hbar^{2}} \sqrt{\frac{i}{k_{m}}} \frac{e^{+i k_{m} r}}{\sqrt{r}} f_{k_{m}, k_{i}}(\theta),
$$

where $f_{k_{m}, k_{i}}(\theta)$ is the scattering amplitude

$$
f_{k_{m}, k_{i}}(\theta)=-\frac{1}{4} \sqrt{\frac{2}{\pi}}<\mathbf{k}_{m}|T(E)| \mathbf{k}_{i}>
$$

with

$$
<\mathbf{k}_{m}|T(E)| \mathbf{k}_{i}>=\sum_{n=0}^{\infty} \int d \mathbf{r}^{\prime} e^{-i \mathbf{k}_{m} \cdot \mathbf{r}^{\prime}} V_{m n}\left(\mathbf{r}^{\prime}\right) \psi_{n i}\left(\mathbf{r}^{\prime}\right) .
$$

The momenta of the initial and final states of the incident (scattered) electron are $\mathbf{k}_{i}$ and $\mathbf{k}_{m}$, respectively, and $\theta$ is the scattering angle between them. It is evident from Eq. (12) and its boundary condition Eq. (19) that the different scattering channels are coupled to each other through the interaction potential $V_{m n}$.

In the above procedure in dealing with the electron scattering through a QD, both the electron-electron exchange and correlation interactions are present in this system. However, a complete correlation effect is difficult to include in a practical calculation. In order to do so, besides an exact solution for the N-electron QD, a full sum over all the intermediate states $n$ in the scattering equation [Eq. (9)] is needed, which is a formidable task in a self-consistent calculation. In an alternative way, the correlation effects can be considered by adding an effective correlation potential in the scattering equation (Joachain, 1975). In the present work, we focus on the exchange effects on the scattering process and limit the sum over $n$ to a few lowest energy levels of the QD. For this reason, we prefer to call the nonlocal interaction potential $V_{m n}^{\text {ex }}$ in Eq. (11) as exchange potential, though the correlation can be partially included.

The differential cross-section (DCS) for a scattering from initial state $i$ (i.e. the incident electron of kinetic energy $E_{i}=\frac{\hbar^{2} k_{i}^{2}}{2 m^{*}}$ and the QD in the state $\varepsilon_{i}$ ) to final state $m$ (i.e. $E_{m}=\frac{\hbar^{2} k_{m}^{2}}{2 m^{*}}$ and the QD in the state $m$ ) is given by

$$
\sigma_{m i}(\theta)=\frac{k_{m}}{k_{i}^{2}}\left|f_{k_{m}, k_{i}}(\theta)\right|^{2} .
$$


The integral cross-section (ICS) which is an energy dependent quantity can be found by

$$
\Gamma_{m i}\left(E_{i}\right)=\int_{0}^{2 \pi} \sigma_{m i}(\theta) d \theta .
$$

When the incident electron is scattered to a state of the same energy and the QD keeps in the same state $(m=i)$, the scattering is called elastic. Otherwise, the scattering is inelastic. A possible scattering is the so-called super-elastic scattering $\left(E_{m}>E_{i}\right)$ where the incident electron is scattered out with a higher energy by an QD initially in an excited state. Because the different scattering channels are coupled to each other, we have to solve the multichannel L-S equation to obtain the scattering probabilities through different channels simultaneously for the same total energy of the system.

\subsection{Partial wave expansion}

In two dimensions the angular momentum basis is given by (Adhikari, 1986),

$$
\Theta_{l}(\phi)=\sqrt{\frac{\kappa_{l}}{2 \pi}} \cos (l \phi)
$$

where $l=0,1,2, \ldots, \kappa_{l}=2$ for $l \neq 0$ and $\kappa_{l}=1$ for $l=0$. In applying the partial wave expansion in the multi-channel scattering problem Eq. (12), we expand all functions, i.e., the incident free electron wavefunction $\varphi_{i}(\mathbf{r})$, the Green's function $G^{(0)}\left(\mathbf{k}_{m}, \mathbf{r}, \mathbf{r}^{\prime}\right)$, and the scattered electron wavefunction $\psi_{m i}(\mathbf{r})$, in the angular momentum basis as follows,

$$
\varphi_{i}(\mathbf{r})=\sum_{l, l^{\prime}=0}^{\infty} \sqrt{\frac{\kappa_{l}}{2 \pi}} i^{l} J_{l}(k r) \delta_{l l^{\prime}} \Theta_{l}\left(\phi_{r}\right) \Theta_{l^{\prime}}\left(\phi_{k}\right),
$$

and

$$
\psi_{m i}(\mathbf{r})=\sum_{l, l^{\prime}=0}^{\infty} \psi_{m i}^{l, l^{\prime}}(k, r) \Theta_{l}\left(\phi_{r}\right) \Theta_{l^{\prime}}\left(\phi_{k}\right),
$$

where $\phi_{r}$ and $\phi_{k}$ are the variables due to expansion on the position $\mathbf{r}$ and momentum $\mathbf{k}$, respectively. The expansion on the Green's function yields the following expression,

$$
G^{(0)}\left(\mathbf{k}_{m}, \mathbf{r}, \mathbf{r}^{\prime}\right)=-\frac{i \pi}{2} \sum_{l=0}^{\infty} \sqrt{\frac{\kappa_{l}}{2 \pi}} J_{l}\left(k_{m} r_{<}\right) H_{l}^{(1)}\left(k_{m} r_{>}\right) \Theta_{l}\left(\phi_{r}\right) \Theta_{l}\left(\phi_{r^{\prime}}\right),
$$

where $k=k_{i}, r_{<}=\min \left(r, r^{\prime}\right), r_{>}=\max \left(r, r^{\prime}\right), J_{l}\left(k_{m} r\right)\left(Y_{l}\left(k_{m} r\right)\right)$ is the Bessel (Neumann) function and $H_{l}^{(1)}\left(k_{m} r\right)=J_{l}\left(k_{m} r\right)+i Y_{l}\left(k_{m} r\right)$ is the Hankel function (Morse \& Feshbach, 1953). Using the partial wave expansion the Lippmann-Schwinger equation can be reduced to a set of radial equations. The radial Lippmann-Schwinger equation corresponding to Eq. (12) is given by,

$$
\psi_{m i}^{l, l^{\prime}}(k, r)=\sqrt{\frac{\kappa_{l}}{2 \pi}} i^{l} J_{l}(k r) \delta_{l l^{\prime}} \delta_{m i}+\sum_{l^{\prime \prime}=0}^{\infty} \sum_{n=0}^{\infty} \int_{0}^{\infty} r^{\prime} d r^{\prime} g_{0}^{l}\left(k_{m}, r, r^{\prime}\right) V_{m n}^{l, l^{\prime \prime}}\left(r^{\prime}\right) \psi_{n i}^{l^{\prime \prime}, l^{\prime}}\left(r^{\prime}\right),
$$

where

$$
g_{0}^{l}\left(k_{m}, r, r^{\prime}\right)=\frac{-i \pi}{2} \sqrt{\frac{\kappa_{l}}{2 \pi}} J_{l}\left(k_{m} r_{<}\right) H_{l}^{(1)}\left(k_{m} r_{>}\right)
$$


and

$$
V_{m n}^{l, l^{\prime \prime}}\left(r^{\prime}\right)=\int_{0}^{2 \pi} d \phi_{r^{\prime}} \Theta_{l}\left(\phi_{r^{\prime}}\right) V_{m n}\left(\mathbf{r}^{\prime}\right) \Theta_{l^{\prime \prime}}\left(\phi_{r^{\prime}}\right)
$$

We see that, when the partial wave method is used, there is a change in the continuum variable $\phi$ to a partial wave $l$. Consequently, the wave function $\psi_{m i}(\mathbf{r})$ becomes a matrix function with elements $\psi_{m i}^{l, l^{\prime}}(k, r)$.

The partial wave expansion for the exchange potential is a little subtle due to its non-locality. Here, we show some details about how the partial wave expansion is applied in this case. We take as an example the exchange potential which couples the channels $n$ and $m$ for a single electron spin-orbital $\alpha$ [see Eq. (11)],

$$
V_{m n}^{\mathrm{ex}}(\mathbf{r}) \psi_{n i}(\mathbf{r})=-\frac{e^{2}}{\epsilon_{0}^{*}} \zeta_{\alpha}^{n}(\mathbf{r}) \int d \mathbf{r}_{1} \zeta_{\alpha}^{m *}\left(\mathbf{r}_{1}\right) \frac{1}{\left|\mathbf{r}-\mathbf{r}_{1}\right|} \psi_{n i}\left(\mathbf{r}_{1}\right) .
$$

The partial wave expansion of the spin-orbital function is given by

$$
\zeta_{\alpha}^{n}(\mathbf{r})=\sum_{l=0}^{\infty} \zeta_{n \alpha}^{l}(r) \Theta_{l}\left(\phi_{r}\right)
$$

The product of two different functions can also be expanded in the angular momentum basis as follows,

$$
\psi_{n i}(\mathbf{r}) \zeta_{\alpha}^{m *}(\mathbf{r})=\sum_{l, l^{\prime}} \Pi_{n i ; m \alpha}^{l, l^{\prime}}(r) \Theta_{l}\left(\phi_{r}\right) \Theta_{l^{\prime}}\left(\phi_{k}\right),
$$

where

$$
\Pi_{n i ; m \alpha}^{l, l^{\prime}}(r)=\sum_{\lambda, \lambda^{\prime}} \frac{\psi_{n i}^{\lambda, l^{\prime}}(k, r) \zeta_{m \alpha}^{\lambda^{\prime} *}(r)}{2 \sqrt{2 \pi}} \sqrt{\frac{\kappa_{\lambda} \kappa_{\lambda^{\prime}}}{\kappa_{l}}}\left(\delta_{l, \lambda+\lambda^{\prime}}+\delta_{l,\left|\lambda-\lambda^{\prime}\right|}\right) .
$$

Using the above relation, we obtain Eq. (30) in the partial wave expansion form,

$$
\begin{aligned}
& V_{m n}^{\mathrm{ex}}(\mathbf{r}) \psi_{n i}(\mathbf{r})=-\frac{e^{2}}{\epsilon_{0}^{*}} \zeta_{\alpha}^{n}(\mathbf{r}) \sum_{l, l^{\prime}} \Theta_{l}\left(\phi_{r}\right) \Theta_{l^{\prime}}\left(\phi_{k}\right) \\
& \times \int_{0}^{\infty} r_{1} d r_{1} \Pi_{n i ; m \alpha}^{l, l^{\prime}}\left(r_{1}\right) \int_{0}^{2 \pi} \frac{\Theta_{l}(\theta) d \theta}{\sqrt{r^{2}+r_{1}^{2}-2 r r_{1} \cos (\theta)}},
\end{aligned}
$$

where $\theta=\phi_{r}-\phi_{r_{1}}$. To solve the angular integral we use the generating function of the Legendre Polynomials (Morse \& Feshbach, 1953),

$$
\frac{1}{\sqrt{r^{2}+r_{1}^{2}-2 r r_{1} \cos (\theta)}}=\sum_{j=0}^{\infty} \frac{r_{<}^{j}}{r_{>}^{j+1}} P_{j}(\cos \theta),
$$

where $r_{<}=\min \left(r, r_{1}\right), r_{>}=\max \left(r, r_{1}\right)$ and $P_{j}(\cos \theta)$ are the Legendre Polynomials. Thus the angular integral that we need to solve is

$$
c_{l, j}=\int_{0}^{2 \pi} d \theta \Theta_{l}(\theta) P_{j}(\cos \theta) .
$$


Substituting the Eqs. (35) and (36) into Eq. (34) we finally obtain the exchange potential

$$
V_{m n}^{\mathrm{ex}}(\mathbf{r}) \psi_{n i}(\mathbf{r})=-\frac{e^{2}}{\epsilon_{0}^{*}} \zeta_{\alpha}^{n}(\mathbf{r}) \sum_{l, l^{\prime}} \Theta_{l}\left(\phi_{r}\right) \Theta_{l^{\prime}}\left(\phi_{k}\right) \sum_{j=0}^{\infty} \int_{0}^{\infty} r_{1} d r_{1} \Pi_{n i ; m \alpha}^{l, l^{\prime}}\left(r_{1}\right) c_{l, j} \frac{r_{<}^{j}}{r_{>}^{j+1}} .
$$

In the numerical calculations, we firstly evaluate the coefficients $c_{l, j}$ given by Eq. (36). Then the integration on $r_{1}$ in Eq. (37) is performed for each iteration in the MCF. Finally we multiply the result by $-\frac{e^{2}}{\epsilon_{0}^{*}} \zeta_{\alpha}^{n}(\mathbf{r})$.

Within the one-channel approximation $(i=m=n=0)$, the calculations can be further simplified by using the concept of phase shift. Considering a central potential $V(r)\left(l=l^{\prime}=\right.$ $\left.l^{\prime \prime}\right)$, Eq. (27) becomes

$$
\psi^{l}(k, r)=\sqrt{\frac{\kappa_{l}}{2 \pi}} i^{l} J_{l}(k r)+\int_{0}^{\infty} r^{\prime} d r^{\prime} g_{0}^{l}\left(k, r, r^{\prime}\right) V\left(r^{\prime}\right) \psi^{l}\left(k, r^{\prime}\right)
$$

where $\psi^{l}(k, r)=\psi_{00}^{l, l}(k, r)$. To define the phase-shift we write the asymptotic form of the above equation as

$$
\psi^{l}(k, r) \underset{r \rightarrow \infty}{\longrightarrow} A_{l} \sqrt{\frac{1}{k r}} \cos \left(k r-\frac{l \pi}{2}-\frac{\pi}{4}-\Delta_{l}\right),
$$

where $\Delta_{l}$ is the phase-shift. Comparing the coefficients of $e^{i k r}$ and $e^{-i k r}$ of Eq. (39) with the asymptotic form of Eq. (38) one can obtain the following relations

$$
A_{l}=2 \sqrt{\frac{\kappa_{l}}{\pi}} i^{l} e^{i \Delta_{l}}
$$

and

$$
e^{i \Delta_{l}} \sin \Delta_{l}=\frac{-\pi}{2 i^{l}} \int_{0}^{\infty} r^{\prime} d r^{\prime} J_{l}\left(k r^{\prime}\right) V\left(r^{\prime}\right) \psi^{l}\left(r^{\prime}\right)
$$

On the other hand, from the definition of the scattering amplitude in Eq. (19), we can express the scattering amplitude $f_{k_{0}, k_{0}}$ in terms of the phase-shift (Adhikari, 1986) $\Delta_{l}$,

$$
f_{k_{0}, k_{0}}(\theta)=2 \sum_{l=0}^{\infty} \sqrt{\frac{\kappa_{l}}{\pi}} e^{i \Delta_{l}} \sin \Delta_{l} \Theta_{l}(\theta)
$$

The corresponding DCS is $\sigma_{00}(\theta)=\left|f_{k_{0}, k_{0}}(\theta)\right|^{2} / k$ and the ICS is given by

$$
\Gamma_{00}=\frac{4}{k} \sum_{l=0}^{\infty} \kappa_{l} \sin ^{2} \Delta_{l} \text {. }
$$

\section{Quantum dot confined in a quasi-one-dimensional structure}

In this section, we study the electron scattering through a QD confined in a quasi-one-dimensional structure. The quantum dot is considered to be confined in the $y$-direction and the incident (scattered) electron moves in the $x$-direction. Far from the QD, the electron is free to propagate in the $x$-direction. In this limit, the Schrödinger equation in the $y$-direction is given by

$$
\left[-\frac{\hbar^{2}}{2 m^{*}} \frac{d^{2}}{d y^{2}}+V_{c}(y)\right] \chi_{n}(y)=\varepsilon_{n} \chi_{n}(y)
$$


We choose the confining potential as being parabolic $V_{c}(y)=\frac{1}{2} m^{*} \omega_{y}^{2} y^{2}$. The solution of equation (44) for this potential is given by (Merzbacher, 1970):

$$
\chi_{n}(y)=\frac{e^{-\frac{y^{2}}{2 l_{y}^{2}}}}{\left(\pi l_{y}^{2}\right)^{1 / 4}} \frac{H_{n}\left(\frac{y}{l_{y}}\right)}{\sqrt{2^{n !}}}
$$

where $H_{n}$ are the Hermite's polinomials (Morse \& Feshbach, 1953), $\varepsilon_{n}=\hbar \omega_{y}(n+1 / 2)$, and $l_{y}=\sqrt{\hbar / m^{*} \omega_{y}}$. The eigenfunctions $\chi_{n}(y)$ are also called transversal modes.

As the basis composed of $\chi_{n}(y)$ is complete, we are able to expand the wave-function in such a system on this basis,

$$
\Psi_{i}(\mathbf{r})=\sum_{n=0}^{\infty} \chi_{n}(y) \psi_{n i}(x),
$$

where $i$ refers to the incident wave-vector. By introducing this result into the Schrödinger equation, multiplying it by $\chi_{m}^{*}(y)$, and integrating in the $y$-direction, we find the following coupled equations:

$$
\left(\frac{\hbar^{2}}{2 m^{*}} \frac{d^{2}}{d x^{2}}+\frac{\hbar^{2} k_{n}^{2}}{2 m^{*}}\right) \psi_{n i}(x)=\sum_{m=0}^{\infty} V_{m, n}(x) \psi_{m i}(x),
$$

where $\frac{\hbar^{2} k^{2}}{2 m^{*}}=\frac{\hbar^{2} k_{n}^{2}}{2 m^{*}}+\varepsilon_{n}$ and

$$
V_{m, n}(x)=\int d y \chi_{m}^{*}(y) V(\mathbf{r}) \chi_{n}(y)
$$

The Green's function is defined as being the solution of the equation:

$$
\left(\frac{\hbar^{2}}{2 m^{*}} \frac{d^{2}}{d x^{2}}+\frac{\hbar^{2} k_{n}^{2}}{2 m^{*}}\right) G_{n}\left(x, x^{\prime}\right)=\delta\left(x-x^{\prime}\right),
$$

which allows to rewrite the solution of Eq. (47) as a Lippmann-Schwinger equation in one-dimension,

$$
\psi_{n i}(x)=\varphi_{n}(x)+\frac{2 m^{*}}{\hbar^{2}} \sum_{m=0}^{\infty} \int d x^{\prime} G_{n}\left(x, x^{\prime}\right) V_{m, n}\left(x^{\prime}\right) \psi_{m i}\left(x^{\prime}\right),
$$

where $\varphi_{n}(x)=\exp \left(i k_{n} x\right) \delta_{n, i} / \sqrt{k_{n}}$. The Green's function to each sub-band in a Q1D system is equal to:

$$
G_{n}\left(x, x^{\prime}\right)=\frac{-i}{2 k_{n}} e^{i k_{n}\left|x-x^{\prime}\right|} .
$$

Because the energy of the incident electron is $\frac{\hbar^{2} k^{2}}{2 m^{*}}=\frac{\hbar^{2} k_{n}^{2}}{2 m^{*}}+\varepsilon_{n}$, there is the possibility of $\varepsilon_{n}>$ $\frac{\hbar^{2} k^{2}}{2 m^{*}}$ and of $k_{n}$ being a pure imaginary number. In such a situation, we must replace $k_{n}$ by $i\left|k_{n}\right|$ in Eq. (50) and the eigenfunctions $\psi_{n i}(x)$ are not localized anymore.

Taking the limit $x \rightarrow \infty$ in Eq. (50), we obtain:

$$
\psi_{n i}(x) \underset{x \rightarrow \infty}{\longrightarrow} \frac{e^{i k_{n} x}}{\sqrt{k_{n}}}\left[\delta_{n i}+\frac{m^{*}}{i \hbar^{2}} \sum_{m=0}^{\infty} \int d x^{\prime} \frac{e^{-i k_{n} x^{\prime}}}{\sqrt{k_{n}}} V_{m, n}\left(x^{\prime}\right) \psi_{m i}\left(x^{\prime}\right)\right] .
$$


The scattering matrix $T$ can be found through the following result

$$
T_{n i}=\sum_{m=0}^{\infty} \int_{-\infty}^{\infty} d x^{\prime} \frac{e^{-i k_{n} x^{\prime}}}{\sqrt{k_{n}}} V_{m, n}\left(x^{\prime}\right) \psi_{m i}\left(x^{\prime}\right) .
$$

Another quantity that we can obtain is transmission probability $t_{n i}$, which by definition satisfies the following equation (Vargiamidis et al., 2003):

$$
\Psi_{i}(\mathbf{r}) \underset{x \rightarrow \infty}{\longrightarrow} \sum_{n=0}^{\infty} t_{n i} \frac{e^{i k_{n} x}}{\sqrt{k_{n}}} \chi_{n}(y) .
$$

Multiplying Eq. (52) by $\chi_{n}(y)$, then adding $n=0$ to $\infty$, and comparing the resulting equation to Eq. (54) we obtain the following expression for $t_{n i}$ :

$$
t_{n i}=\delta_{n i}+\frac{m^{*}}{i \hbar^{2}} \sum_{m=0}^{\infty} \int_{-\infty}^{\infty} d x^{\prime} \frac{e^{-i k_{n} x^{\prime}}}{\sqrt{k_{n}}} V_{m, n}\left(x^{\prime}\right) \psi_{m}\left(x^{\prime}\right) .
$$

So we can relate the matrix $T$ with the scattering transmission probability $t_{n i}$ by:

$$
t_{n i}=\delta_{n i}+\frac{m^{*}}{i \hbar^{2}} T_{n i}
$$

These quantities are useful to determine the conductance (Fisher \& Lee, 1981; Imry \& Landauer, 1999). In the Q1D system with multiple scattering channels, the conductance can be obtained by using the Landauer-Büttiker equation (Buttiker et al., 1985; Landauer, 1957; 1970; 1975),

$$
G=\frac{e^{2}}{\pi \hbar} \operatorname{Tr}\left(t t^{\dagger}\right),
$$

where $t$ is the matrix whose elements are exactly given by Eq. (55).

\section{Applications and numerical results}

In the previous sections, we presented a theoretical model that describes the quantum scattering through a quantum dot with $N$-electrons confined. However, we apply this model to the case where only one electron is confined in the quantum dot. Although this is the simplest case, it reveals basic information for a more complicated system. In this section, we describe the details of this particular case considering the elastic and inelastic scattering in the 2D system in sub-sections 5.1 and 5.2, respectively. The scattering through a confined QD in the Q1D system will be discussed in sub-section 5.3.

\subsection{Elastic scattering}

Here we describe in details how the elastic scattering can be accounted for. To do so, we start by considering the electron in the ground state of energy $\varepsilon_{1}$. The total Hamiltonian for this system (incident electron + confined electron) is given by:

$$
H\left(\mathbf{r}_{1}, \mathbf{r}_{2}\right)=\frac{-\hbar^{2} \nabla_{2}^{2}}{2 m^{*}}+V_{Q D}\left(\mathbf{r}_{2}\right)+H_{Q D}\left(\mathbf{r}_{1}\right)+V\left(\mathbf{r}_{1}, \mathbf{r}_{2}\right)
$$

where $H_{Q D}$ is the QD Hamiltonian and $V\left(\mathbf{r}_{1}, \mathbf{r}_{2}\right)$ is the Coulomb interaction potential between the pair of electrons. The total wave function should be written as linear combination of 
Slater determinants, as shown in sub-section 2.2. There are four possible combinations for two electrons,

$$
\begin{aligned}
& \left|\overline{\Phi^{1}}, \overline{\psi_{11}}>=\frac{1}{\sqrt{2}}\right| \begin{array}{ll}
\Phi^{1}\left(\mathbf{r}_{1}\right) \beta(1) & \psi_{11}\left(\mathbf{r}_{1}\right) \beta(1) \\
\Phi^{1}\left(\mathbf{r}_{2}\right) \beta(2) & \psi_{11}\left(\mathbf{r}_{2}\right) \beta(2)
\end{array} \mid, \\
& \left|\Phi^{1}, \psi_{11}>=\frac{1}{\sqrt{2}}\right| \begin{array}{ll}
\Phi^{1}\left(\mathbf{r}_{1}\right) \alpha(1) & \psi_{11}\left(\mathbf{r}_{1}\right) \alpha(1) \\
\Phi^{1}\left(\mathbf{r}_{2}\right) \alpha(2) & \psi_{11}\left(\mathbf{r}_{2}\right) \alpha(2)
\end{array} \mid, \\
& \left|\Phi^{1}, \overline{\psi_{11}}>=\frac{1}{\sqrt{2}}\right| \begin{array}{ll}
\Phi^{1}\left(\mathbf{r}_{1}\right) \alpha(1) & \psi_{11}\left(\mathbf{r}_{1}\right) \beta(1) \\
\Phi^{1}\left(\mathbf{r}_{2}\right) \alpha(2) & \psi_{11}\left(\mathbf{r}_{2}\right) \beta(2)
\end{array} \mid,
\end{aligned}
$$

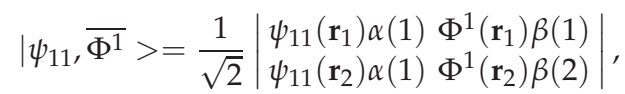

where $\Phi^{1}(\mathbf{r})$ is the wave function of the confined electron in the QD, $\alpha(i)$ and $\beta(i)$ correspond to spin-up $(\uparrow)$ and spin-down $(\downarrow)$, respectively. The index $(i)$ denotes to which electron the spin refers to.

Because the total Hamiltonian (Eq. (58)) commutes with the total spin operator $\left(S^{2}\right)$ and its component in the $z$-direction $\left(S_{z}\right)$, the Hamiltonian eigenfunctions must be eigenfunctions of both $S_{z}$ and $S^{2}$. The first two determinants of Slater in equations (59 and 60) are eigenfunctions of $S_{z}$ and $S^{2}$, but the equations (61 and 62) are not eigenfunctions of $S^{2}$. Thus, we have to construct linear combinations between these Slater determinants (Eqs. (61 and 62)) in order to obtain eigenfunctions of $S_{z}$ and $S^{2}$. These combinations can be written as follows:

$$
\begin{aligned}
& \left.\left|\Psi^{\mathcal{S}}\right\rangle=\frac{1}{\sqrt{2}}\left[\left|\Phi^{1}, \overline{\psi_{11}}>+\right| \psi_{11}, \overline{\Phi^{1}}\right\rangle\right]= \\
& =\frac{1}{\sqrt{2}}\left[\psi_{11}\left(\mathbf{r}_{1}\right) \Phi^{1}\left(\mathbf{r}_{2}\right)+\psi_{11}\left(\mathbf{r}_{2}\right) \Phi^{1}\left(\mathbf{r}_{1}\right)\right]\left(\frac{|\downarrow, \uparrow>-| \uparrow, \downarrow>}{\sqrt{2}}\right)
\end{aligned}
$$

and

$$
\begin{aligned}
& \mid \Psi^{t}>=\frac{1}{\sqrt{2}}\left[\left|\Phi^{1}, \overline{\psi_{11}}>-\right| \psi_{11}, \overline{\Phi^{1}}>\right]= \\
& =\frac{1}{\sqrt{2}}\left[\psi_{11}\left(\mathbf{r}_{1}\right) \Phi^{1}\left(\mathbf{r}_{2}\right)-\psi_{11}\left(\mathbf{r}_{2}\right) \Phi^{1}\left(\mathbf{r}_{1}\right)\right]\left(\frac{|\downarrow, \uparrow>+| \uparrow, \downarrow>}{\sqrt{2}}\right) .
\end{aligned}
$$

Equation (63) corresponds to the wave function of the singlet state and Equation (59, 60 and 64) correspond to wave functions of the triplet states. Since the Hamiltonian (Eq. (58)) does not have a explicit spin-dependent potential, the state of total spin is conserved before and after the collision. In such a way, the total wave function of the system (incident electron + confined electron) can be written as:

$$
\Psi\left(\mathbf{r}_{1}, \mathbf{r}_{2}\right)=\Phi^{1}\left(\mathbf{r}_{1}\right) \psi_{11}\left(\mathbf{r}_{2}\right) \pm \Phi^{1}\left(\mathbf{r}_{2}\right) \psi_{11}\left(\mathbf{r}_{1}\right),
$$

where the positive (negative) sign refers to the spin singlet (triplet) state. In order to determine the potential for the scattered electron, we have to calculate the following equation:

$$
<\Phi^{1}\left(\mathbf{r}_{1}\right)\left|H\left(\mathbf{r}_{1}, \mathbf{r}_{2}\right)\right| \Psi\left(\mathbf{r}_{1}, \mathbf{r}_{2}\right)>=E<\Phi^{1}\left(\mathbf{r}_{1}\right) \mid \Psi\left(\mathbf{r}_{1}, \mathbf{r}_{2}\right)>,
$$

where

$$
E=\varepsilon_{1}+\frac{\hbar^{2} k_{1}^{2}}{2 m^{*}}
$$


The left hand side of Eq. (66) can be rewritten as:

$$
<\Phi^{1}|H| \Psi>=<\Phi^{1}\left|H_{Q D}^{1}\right| \Psi>+<\Phi^{1}\left|H_{Q D}^{2}\right| \Psi>+<\Phi^{1}\left|V^{1,2}\right| \Psi>,
$$

where the superscript is related to each electron the operator is operating on. The first term of Equation (68) is equal to:

$$
<\Phi^{1}\left|H_{Q D}^{1}\right| \Psi>=\varepsilon_{1}\left[<\Phi^{1}\left|\Phi^{1}>\psi_{11} \pm<\Phi^{1}\right| \psi_{11}>\Phi^{1}\right] .
$$

While the second term of Eq. (68) is given by

$$
<\Phi^{1}\left|H_{Q D}^{2}\right| \Psi>=<\Phi^{1}\left|\Phi^{1}>H_{Q D}^{2} \psi_{11} \pm \varepsilon_{1}<\Phi^{1}\right| \Phi^{1}>\psi_{11} .
$$

The third term of Eq. (68) can be written as:

$$
<\Phi^{1}\left|V^{1,2}\right| \Psi>=<\Phi^{1}\left|V^{1,2}\right| \Phi^{1}>\psi_{11} \pm<\Phi^{1}\left|V^{1,2}\right| \psi_{11}>\Phi^{1} .
$$

By substituting Eqs.(69, 70, and 71) into Eq. (68), we obtain the following result:

$$
\begin{aligned}
& \left(H_{Q D}^{2}-\frac{\hbar^{2} k_{1}^{2}}{2 m^{*}}\right) \psi_{11}+<\Phi^{1}\left|V^{1,2}\right| \Phi^{1}>\psi_{11} \pm \\
& \pm\left(<\Phi^{1}\left|V^{1,2}\right| \psi_{11}>\Phi^{1}+\left(\varepsilon_{1}-\frac{\hbar^{2} k_{1}^{2}}{2 m^{*}}\right)<\Phi^{1} \mid \psi_{11}>\Phi^{1}\right)=0 .
\end{aligned}
$$

The previous equation can be further simplified as

$$
-\frac{\hbar^{2}}{2 m^{*}}\left(\nabla^{2}+k_{1}^{2}\right) \psi_{11}(\mathbf{r})+\left[V^{\text {st }}(\mathbf{r}) \pm V^{\mathrm{ex}}(\mathbf{r})\right] \psi_{11}(\mathbf{r})=0,
$$

where

$$
V^{\mathrm{st}}(\mathbf{r})=<\Phi^{1}\left|V^{1,2}\right| \Phi^{1}>+V_{Q D}(\mathbf{r}),
$$

and

$$
V_{11}^{\mathrm{ex}}(\mathbf{r}) \psi_{11}(\mathbf{r})=\Phi^{1}(\mathbf{r})\left[<\Phi^{1}\left|V^{1,2}\right| \psi_{11}>+\left(\varepsilon_{1}-\frac{\hbar^{2} k_{1}^{2}}{2 m^{*}}\right)<\Phi^{1} \mid \psi_{11}>\right] .
$$

Finally, the Lippmann-Schwinger equation corresponding to Eq. (73) is given by

$$
\left.\psi_{11}(\mathbf{r})=\varphi_{1}(\mathbf{r})+\int d \mathbf{r}^{\prime} G^{(0)} \mathbf{k}_{1}, \mathbf{r}, \mathbf{r}^{\prime}\right)\left[V^{s t}\left(\mathbf{r}^{\prime}\right) \pm V^{e x}\left(\mathbf{r}^{\prime}\right)\right] \psi_{11}\left(\mathbf{r}^{\prime}\right),
$$

which can be numerically solved by the method of continuous fractions.

From Eq. (76), we observe that the exchange potential is different when the two electrons form a singlet spin state (plus sign) or triplet spin state (minus sign). In order to calculate the spin-dependent scattering, we have to calculate separately the scattering considering the singlet state and the triplet state. Moreover, the cross sections are given by

$$
\sigma_{11}^{s}(\theta)=\frac{1}{k_{1}}\left|f_{11}^{s}(\theta)\right|^{2},
$$


for the singlet state, and

$$
\sigma_{11}^{t}(\theta)=\frac{1}{k_{1}}\left|f_{11}^{t}(\theta)\right|^{2}
$$

for the triplet state, where

$$
\begin{aligned}
& f_{11}^{s}(\theta)=-\frac{1}{4} \sqrt{\frac{2}{\pi}} \int d^{2} \mathbf{r}^{\prime} e^{-i \mathbf{k}_{1}^{\prime} \cdot \mathbf{r}^{\prime}}\left[V^{s t}\left(\mathbf{r}^{\prime}\right)+V^{e x}\left(\mathbf{r}^{\prime}\right)\right] \psi_{11}\left(\mathbf{r}^{\prime}\right), \\
& f_{11}^{t}(\theta)=-\frac{1}{4} \sqrt{\frac{2}{\pi}} \int d^{2} \mathbf{r}^{\prime} e^{-i \mathbf{k}_{1}^{\prime} \cdot \mathbf{r}^{\prime}}\left[V^{s t}\left(\mathbf{r}^{\prime}\right)-V^{e x}\left(\mathbf{r}^{\prime}\right)\right] \psi_{11}\left(\mathbf{r}^{\prime}\right) .
\end{aligned}
$$

A quantity that we can obtain is the spin-unpolarized cross section (su-ICS), which is given by the statistical average of possible configurations, i.e.,

$$
\sigma_{11}^{s u}(\theta)=\frac{1}{4 k_{1}}\left(\left|f_{11}^{s}(\theta)\right|^{2}+3\left|f_{11}^{t}(\theta)\right|^{2}\right)
$$

where the factor three that multiplies the squared modulus of the scattering amplitude of the triplet state is due to the existence of three different triplet states, which are scattered with equal probability. Another quantity that we can extract from the calculation is the spin-flip (sf) cross-section (da Paixão et al., 1996; Hegemann et al., 1991), which measures the probability of the incident electron changes its spin after being scattered,

$$
\sigma_{11}^{s f}(\theta)=\frac{1}{4 k_{1}}\left|f_{11}^{t}(\theta)-f_{11}^{s}(\theta)\right|^{2}
$$

In the last expression the factor three does not appear because only one of the triplet states can change its spin $\frac{1}{\sqrt{2}}(|\uparrow \downarrow>+| \downarrow \uparrow>)$.

\subsection{Multi-channel scattering}

A very important process that we can study by using the previous formalism is the multi-channel scattering, which reveals the probability of an incident electron to promote an excitation or the decay of electrons within the quantum dot. A priori the number of channels of excitation and decay are infinite, but obviously when doing calculations, this number must be truncated. In the case of the parabolic potential of a 2D quantum dot, the first excited energy level is doubly degenerate with an angular momentum $l= \pm 1$. To consider the possible channels of scattering described by the ground state $\epsilon_{1}$ and by the first excited state $\epsilon_{2}$, we must consider three coupled channels because the degeneracy of the first excited energy level must be included in the calculation. As the probability of finding the electron in the ground state initially is higher and as the excitation to the first excited state is more likely, we consider only three coupled channels. The calculation details can be found in Ref.(Castelano, 2006). Here we will present some numerical results in Section 6.2.

\subsection{Scattering through the QD confined in the Q1D structure}

As already discussed in Section 4, the Lippmann-Schwinger equation for the confined QD includes several sub-bands. However, as a first example, we consider only the lowest 
transversal sub-band due to the confinement in the $y$-direction. Thus, the L-S equation for a single sub-band is given by:

$$
\psi_{1}(x)=\frac{e^{i k_{1} x}}{\sqrt{k_{1}}}+\frac{2 m^{*}}{\hbar^{2}} \int d x^{\prime} G_{1}\left(x, x^{\prime}\right) V_{1,1}\left(x^{\prime}\right) \psi_{1}\left(x^{\prime}\right),
$$

where $\frac{\hbar^{2} k^{2}}{2 m^{*}}=\frac{\hbar^{2} k_{1}^{2}}{2 m^{*}}+\varepsilon_{1}$. The potential and Green's function for the one sub-band case are respectively given by

$$
V_{1,1}(x)=\int d y \chi_{1}^{*}(y) V(\mathbf{r}) \chi_{1}(y)
$$

and

$$
G_{1}\left(x, x^{\prime}\right)=\frac{-i}{2 k_{1}} e^{i k_{1}\left|x-x^{\prime}\right|} .
$$

We also consider only one confined electron in the QD. The electron wave function of the ground state of the QD can be approximated as

$$
\Phi_{1}(x, y)=\frac{1}{\sqrt{\pi l_{x} l_{y}}} \exp \left(-\frac{x^{2}}{2 l_{x}^{2}}-\frac{y^{2}}{2 l_{y}^{2}}\right),
$$

where $l_{x}=\sqrt{\hbar / m^{*} \omega_{x}}$ and $l_{y}=\sqrt{\hbar / m^{*} \omega_{y}}$. Here we consider the QD confining potential in the $x$-direction as a finite parabolic one with confinement frequency $\omega_{x}$. The calculation of the exchange potential is more complicated in the Q1D system. However, when we use the wave function of the harmonic oscillator, we can partially obtain analytical expressions for the exchange potential.

Just as in the elastic scattering in the $2 \mathrm{D}$ case without extra confinement, the exchange potential is different when the two electrons form a singlet or a triple spin state. So, we have to calculate separately the scattering for the different spin sates. The $T$ matrices can be obtained by the following equations:

$$
T_{11}^{s}=\int_{-\infty}^{\infty} d x^{\prime} \frac{e^{-i k_{1} x^{\prime}}}{\sqrt{k_{1}}}\left[V_{1,1}^{s t}\left(x^{\prime}\right)+V_{1,1}^{e x}\left(x^{\prime}\right)\right] \psi_{11}\left(x^{\prime}\right),
$$

and

$$
T_{11}^{t}=\int_{-\infty}^{\infty} d x^{\prime} \frac{e^{-i k_{1} x^{\prime}}}{\sqrt{k_{1}}}\left[V_{1,1}^{s t}\left(x^{\prime}\right)-V_{1,1}^{e x}\left(x^{\prime}\right)\right] \psi_{11}\left(x^{\prime}\right),
$$

where

$$
V_{1,1}^{s t}(x)=\int d y \chi_{1}^{*}(y) V^{s t}(\mathbf{r}) \chi_{1}(y)
$$

and

$$
V_{1,1}^{e x}(x) \psi_{11}(x)=\int d y \chi_{1}^{*}(y) V^{e x}(\mathbf{r}) \chi_{1}(y) \psi_{11}(x) .
$$

The static potential $V_{1,1}^{s t}(x)$ and the exchange potential $V_{1,1}^{e x}(x)$ are analog to the Eqs. (10) and (11). The transmission probability for the electrons behaving as singlet and triplet states can also be obtained, see Section 4. 


\section{Results and analysis}

In this section, we present the numerical results of scattering of the incident electron through a quantum dot containing just one confined electron. The first step we have to do is to calculate the eigenfunctions and the eigenenergies of the Hamiltonian Eq. (4) for $N=1$, which has the following form:

$$
\left[-\frac{\hbar^{2}}{2 m^{*}} \nabla^{2}+V_{Q D}(r)\right] \Phi^{n}=H_{Q D} \Phi^{n}=\varepsilon_{n} \Phi^{n},
$$

where

$$
V_{Q D}(r)=\left\{\begin{array}{cc}
\frac{1}{2} m^{*} \omega_{0}^{2}\left(r^{2}-R_{0}^{2}\right), & r<R_{0} \\
0, & r>R_{0} .
\end{array}\right.
$$

Usually, the QD is modeled by an infinite parabolic potential $V_{Q D}(r)=\frac{1}{2} m^{*} \omega_{0}^{2} r^{2}$. However,

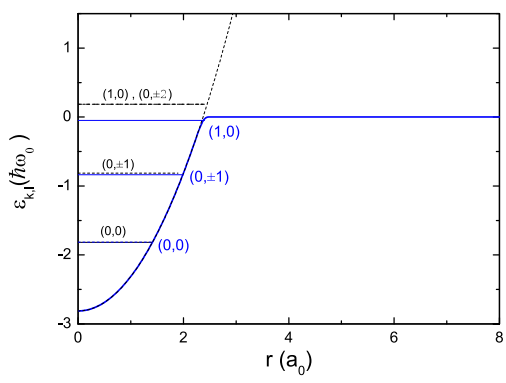

(a) Energy Levels

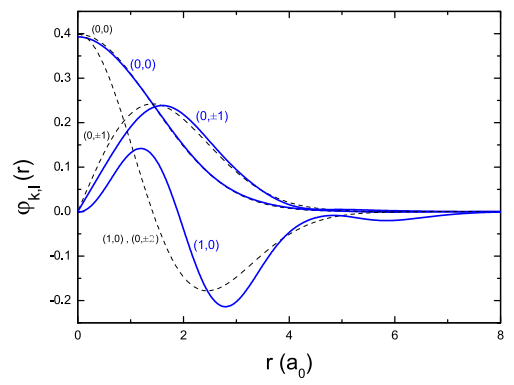

(b) Wave Functions

Fig. 2. (a) Energy levels and (b) wave functions of the infinite (black dashed curves) and finite (blue solid curves) parabolic potential for $R_{0}=2.37 a_{0}$. The indexes $(k, l)$ denote the radial and angular quantum numbers, respectively.

we are dealing with the scattering processes of an incident electron through the QD, we must employ a potential that goes to zero at the infinity. Therefore, we use the finite parabolic potential (Eq. (92)) as the QD potential. We solve Eq. (91) by expanding the wave function $\Phi^{n}$ in the Fock-Darwin basis (Darwin, 1930; Fock, 1928). The eigenenergies and eigenfunctions are determined by diagonalizing the matrix within the Fock-Darwin basis. Figure 2 (a) compares the energy levels of the infinite and finite parabolic potential. From Figs. 2 (a) and (b) we can see that the ground state $(0,0)$ and the first excited state $(0, \pm 1)$ of the finite parabolic potential are not very different from the infinite parabolic potential for $R_{0}=2.37 a_{0}$, where $a_{0}=\sqrt{\hbar / m^{*} \omega_{0}}$. However, the state $(1,0)$ is quite different for the two potentials. We also see that the finite parabolic potential with $R_{0}=2.37 a_{0}$ supports three discrete levels only.

\subsection{Elastic scattering}

The differential cross sections (DCS) for elastic scattering are shown in Figs. 3 (a), (b), and (c) for different incident electron energies $E_{0}=\hbar^{2} k_{0}^{2} / 2 m^{*}=0.6 \mathrm{meV}, 1.7 \mathrm{meV}$, and $4.2 \mathrm{meV}$, respectively. In order to understand the role of the electron spin in the scattering, we compare the DCS due to the static potential (blue solid curve) with the spin unpolarized scattering 

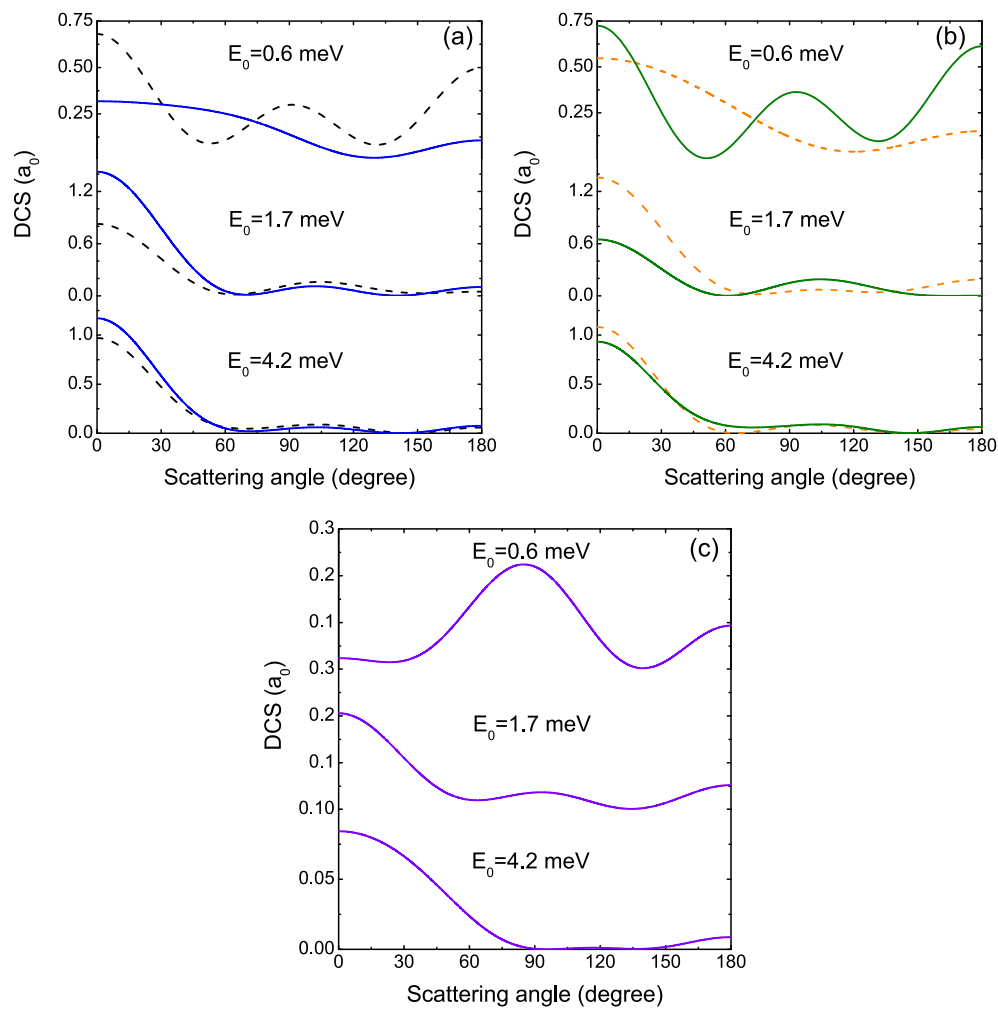

Fig. 3. The elastic DCS's obtained within the one-channel model for electron scattering by the one-electron QD of $\hbar \omega_{0}=5 \mathrm{meV}$ and $r_{0}=35 \mathrm{~nm}$. The incident electron energies are indicated in the figures. (a) The spin-unpolarized DCS with (the dashed curves) and without (the solid curves) the exchange potential; (b) The DCS due to the singlet state (the dashed curves) and the triplet state (the solid curves); and (c) The spin-flip DCS.

(black dashed curve) in Figure 3 (a). It is evident that the electron spin is of significant contribution to low energy $\left(E_{0}=0.6 \mathrm{meV}\right)$ and/or small scattering angles. The exchange effect on the scattering originates from the two different coupling states between the incident and the QD electron (i.e., the singlet and the triplet states) during the collision. The difference due to the spin states for low-energy and small scattering angles is evident in Figure 3 (b), which compares the DCS of the singlet (orange dashed curve) to that of the triplet (green solid curve) state. For higher energies $E_{0}=1.7 \mathrm{meV}$ and $E_{0}=4.2 \mathrm{meV}$, the DCS for singlet and for triplet are similar. We observe that the spin-flip DCS in Figure 3 (c) reaches to maximum for angles close to $\pi / 2$ for $E_{0}=0.6 \mathrm{meV}$, while for $E_{0}=1.7 \mathrm{meV}$ and $E_{0}=4.2 \mathrm{meV}$ its maximum appears at angles close to zero.

Figure 4 (a) shows the integral cross section (ICS) for the elastic scattering by static potential (blue solid curve) and spin unpolarized (black dashed curve). Once again, the importance of the dependence on the spin emerges at low energies. The ICS without including the exchange potential is very different from that considering the electron exchange effects for 

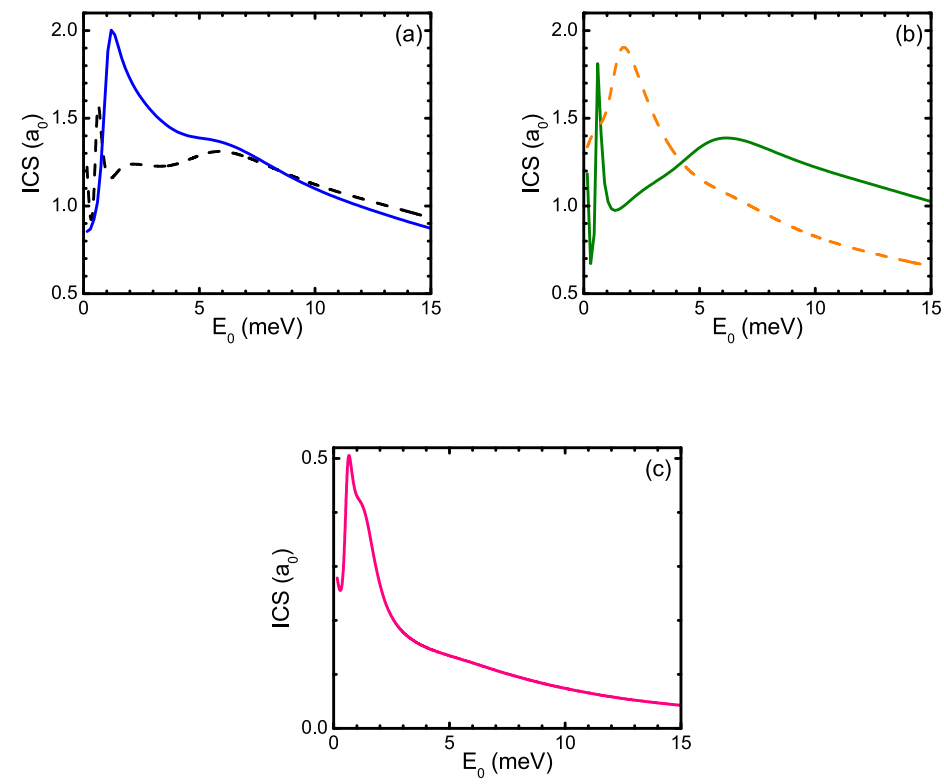

Fig. 4. The elastic ICS as a function of the incident electron energy. (a) The spin-unpolarized ICS with (the dashed curves) and without (the solid curves) the exchange potential; (b) The ICS due to the singlet state (the dashed curves) and the triplet state (the solid curves); and (c) The spin-flip ICS. The parameters for the QD are $\hbar \omega_{0}=5 \mathrm{meV}$ and $r_{0}=35 \mathrm{~nm}$

small incident electron energy. However, the ICS is dominated by the static potential at higher energies.

The integral cross section for the singlet (orange dashed curve) and for the triplet (green solid curve) are shown in Figure 4 (b). Note that in the both cases, as well as in the ICS of the static potential (Fig. 4 (a)), a resonant scattering occurs. These resonances can be explained by the analyzing the phase shifts as shown in Figs. 6 (a), (b), and (c). Generally, the phase shifts are functions that smoothly vary as a function of energy. However, under certain circumstances a sudden change of the phase shifts happens in a energy range and a dramatic change in the cross section takes places for these energies, as can be verified by Eq.(43). A physical explanation to this fact can be found when we consider the Schrödinger equation for a central potential, in the basis of angular momentum (equivalent to Eq. (38)),

$$
\left[\frac{1}{r} \frac{d}{d r}\left(r \frac{d}{d r}\right)+\frac{l^{2}}{r^{2}}+\frac{2 m^{*}}{\hbar^{2}} V(r)\right] \psi^{l, l}(k, r)+k^{2} \psi^{l, l}(k, r)=0 .
$$

We may identify in Eq. (93) an effective potential $V_{e f}=\frac{l^{2}}{r^{2}}+\frac{2 m^{*}}{\hbar^{2}} V(r)$, for each different angular momentum $l$. Figure 5 shows this effective potential as a function of $r$ for $V(r)=$ $V_{Q D}(r)$. By assuming that there is a metastable state with energy $E_{r}$, as sketched in Figure 5 , one can prove that when the electron energy $E_{0}$ reaches $E_{r}$, a rapidly varying phase shift 


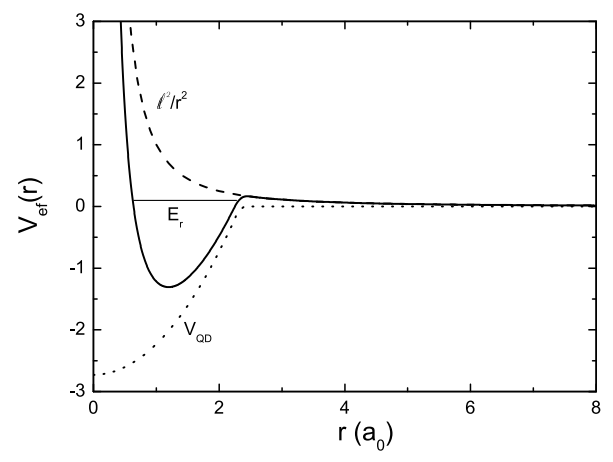

Fig. 5. The effective potential as a function of $r$ is represented by the solid curve. Also, we plot the QD potential (dotted curve) and centrifugal barrier (dashed curve).

occurs and a resonance appears in the ICS (Joachain, 1975). Because this resonance depends on the potential's shape $V(r)$, it is usually called shape resonance.

So, the resonance that appears in the ICS for the static potential (Fig. 4 (a)) with energy $E_{0}=$ $1.22 \mathrm{meV}$ corresponds to the rapid fluctuation of the phase shift $\Delta_{2}$ shown in Figure 6 (a). In this case $\Delta_{2} \approx \pi / 2$ for the value of the energy $E_{0}=1.22 \mathrm{meV}$. We also note the appearance of another broader resonance at $E_{0}=6.14 \mathrm{meV}$, which corresponds to the rapid increasing of $\Delta_{3}$ seen in Figure 6 (a). The singlet resonance $\left(E_{0}=1.72 \mathrm{meV}\right)$ and the triplet resonance $\left(E_{0}=0.57\right.$ $\mathrm{meV}$ ) shown in Fig. 4 (b) are also resulting from the rapidly varying $\Delta_{2}$ plotted in Figs.6 (b) and (c).

In Figure 4 (c) we present the integral elastic spin-flip cross section. The spin-flip cross section depends on the scattering amplitudes of the singlet and the triplet as shown by equation (82). As the ICS modulus (the square of the scattering amplitudes) of singlet and triplet states are very distinct at small energies, the spin-flip cross section is of maximum at the same energy range, as shown in Figure 4 (c).

Figure (7) shows the elastic ICS varying the size of the QD, for the scattering by the static potential (Coulomb without exchange). When the radius $R_{0}$ is increased, the potential of QD becomes more negative and the resonance energy $E_{r}$ (Fig. 5) decreases. Thus, we see that the resonance peak in Fig. (7) shifts to lower energy values when $R_{0}$ increases. Therefore, the potential becomes deeper and the metastable state $E_{r}$ decreases, consequently, the shape resonance shifts to lower energies. The shape resonance may disappear when the radius is further increased. In this situation, the state $E_{r}$ becomes a real bound state instead of a metastable sate.

\subsection{Multi-channel scattering}

When we consider the three-channel scattering, we have nine possibilities of scattering, i.e., the incident electron has initially energy $E_{0}=\hbar^{2} k_{i}^{2} / 2 m^{*}$ and can be scattered with the energy 


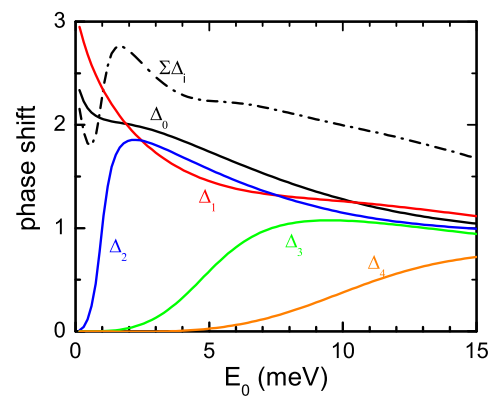

(a) Static potential

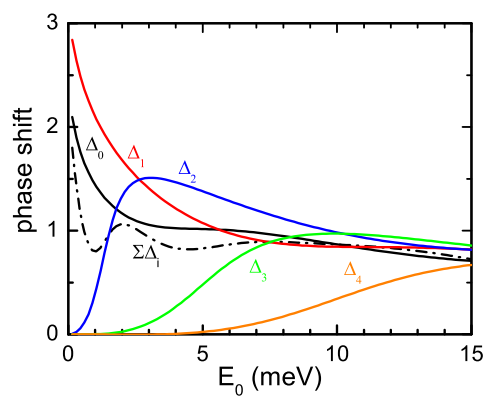

(b) Singlet state

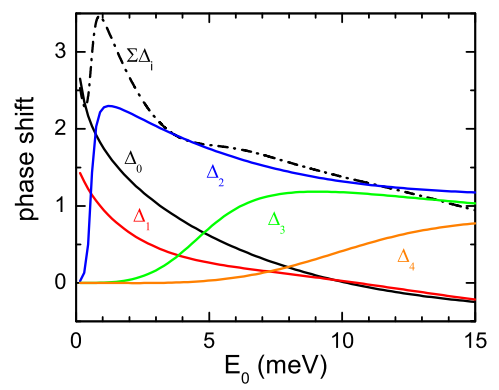

(c) Triplet state.

Fig. 6. The phase shift $\Delta_{l}$ as a function of the incident electron energy for the partial waves with angular momentum $l=0,1,2,3$, and 4 .

$\frac{\hbar^{2} k_{f}^{2}}{2 m}=\frac{\hbar^{2} k_{i}^{2}}{2 m}+\varepsilon_{i}-\varepsilon_{f}$, with $i$ and $f=1,2$, and 3. If $\varepsilon_{f}=\varepsilon_{i}$ the scattering is elastic and if $\varepsilon_{f}>\varepsilon_{i}$ there is a excitation. Finally, if $\varepsilon_{f}<\varepsilon_{i}$ there is a decay.

In our case, as $\varepsilon_{2}=\varepsilon_{3}$ the probability of exciting or decaying for either of one of these two states is exactly the same. Thus, we calculate the cross section considering elastic and inelastic scattering $\varepsilon_{1} \rightarrow \varepsilon_{2}$. Fig. 8 shows the ICS for the elastic channel (a) and for the excitation channel (b). The black (blue) solid curve represents the spin-unpolarized potential (static), while the dashed curves represent the respective ICS when only one channel is considered. For $E_{0} \approx 7 \mathrm{meV}$, we notice that the ICS for the excitation channel in Fig. 8 (b) has a maximum, while the elastic channel in Fig. 8(a) exhibits a minimum, which is obvious from the probability current conservation. For $E_{0}>8 \mathrm{meV}$, the behavior of the ICS shown in Fig. 8 (a) is very similar to the results considering the elastic scattering (dashed curves).

In Figure 9, the ICS is shown for scattering by three-channels, where the green (orange) solid curve represents the scattering by the potential of the triplet (singlet) and the dashed curves represent their ICS when only one channel is considered. In the case of scattering for the triplet state, we verify that when $E_{0}>9 \mathrm{meV}$ the elastic scattering (Figure 9 (a)) for three-channels is equal to one-channel and therefore, the probability of excitation is practically null (Figure 


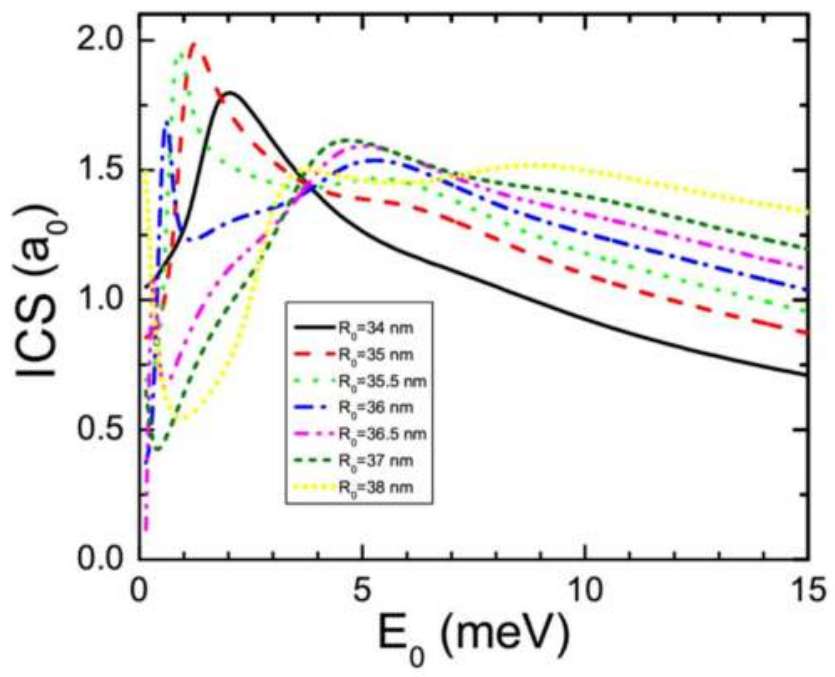

Fig. 7. The elastic ICS as a function of the incident electron energy, considering the static potential for different sizes $\left(R_{0}\right)$ of the QD with $\hbar \omega_{0}=5 \mathrm{meV}$.

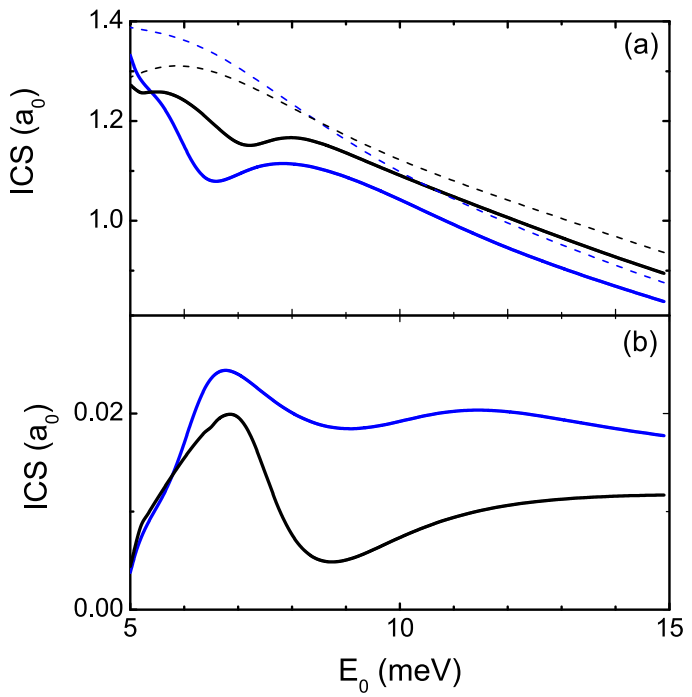

Fig. 8. The ICS for the three-channel scattering as a function of electron energy $\left(R_{0}=35 \mathrm{~nm}\right.$ and $\hbar \omega_{0}=5 \mathrm{meV}$ ). (a) Elastic channel and (b) excitation channel from the ground state to the first excited state $(l= \pm 1)$. Black (blue) curve shows the ICS for the spin-unpolarized (static) scattering. Dashed curves are the respective ICS within the one-channel scattering approximation. 


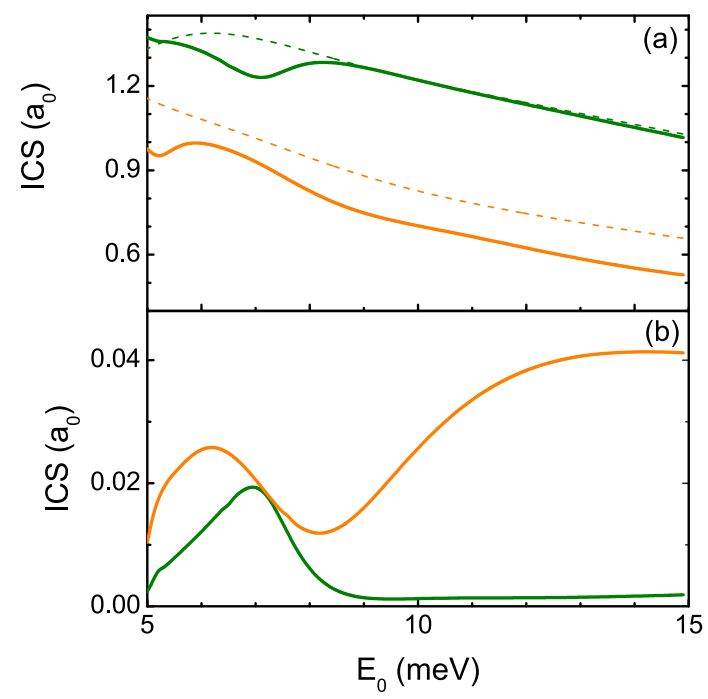

Fig. 9. The same as Fig.8 but now the orange (green) curve shows the ICS for the triplet (singlet) spin state. Dashed curves are the respective ICS within the one-channel scattering approximation.

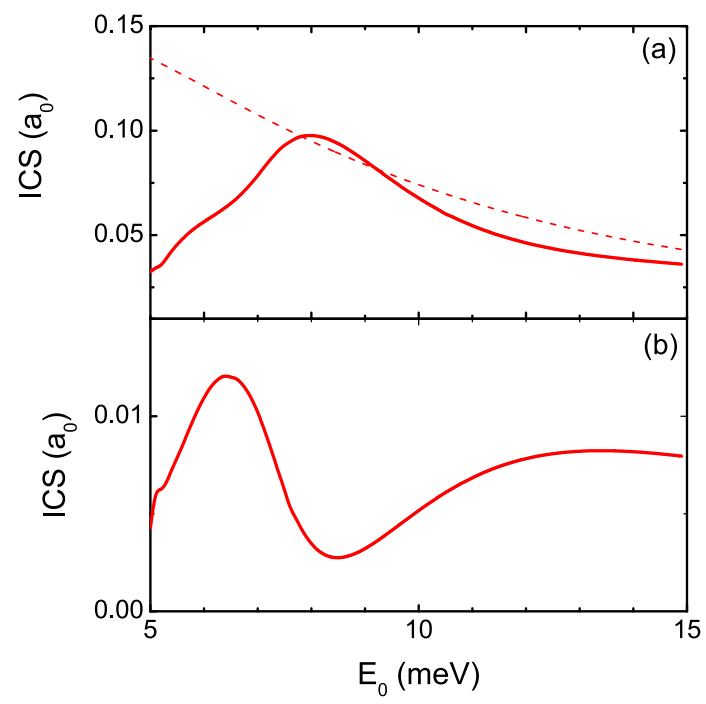

Fig. 10. The same as Fig. 8 but now for the spin-flip ICS. The dashed curve is the respective spin-flip ICS within the one-channel approximation.

9 (b)). In the case of the singlet state, the behavior is contrary, i.e., when $E_{0}>9 \mathrm{meV}$ the excitation probability begins to increase (Fig 9 (b)), thus showing that the scattering may be completely different depending on the spin state of electrons. 
In Figure 10, the spin-flip ICS is shown considering three-channels of scattering, where the solid curve represents the scattering from (a) elastic and (b) excitation channel. The dashed curve represents the spin-flip ICS considering only one channel of scattering. The spin-flip ICS for one-channel presents a maximum for $E_{0} \approx 8 \mathrm{meV}$ and a minimum in the same energy range for the excitation channel. We also found that for $E_{0}>9 \mathrm{meV}$, the spin-flip cross section of three-channels is similar to the elastic scattering (dashed curve).

\subsection{Scattering in the quasi-one-dimensional system}

In this section, we apply the MCF to solve the Lippmann-Schwinger equation for the electron scattering through the QD confined in the Q1D structure. The convergency of MCF is very accurate in this case and achieves a precision of $10^{-4}$ for the transmission probability in approximately 20 interactions. To probe our numerical method, we consider one electron confined in the QD with radius $R_{0}=45 \mathrm{~nm}$. Moreover, two different cases for confined potential in $x$-direction with $\hbar \omega_{x}=5 \mathrm{meV}$ and $\hbar \omega_{x}=3 \mathrm{meV}$ are tested. The obtained results are shown in Fig. 11. In both cases, the screening length is fixed as $\lambda^{-1}=l_{x}$ and the confinement frequency the $y$-direction is set different from that in the $x$-direction with $\omega_{y}=1.7 \omega_{x}$. In Fig. 11 (a) and (b), we plot the transmission probability as a function of the incident electron energy assuming different scattering situations: (i) the QD potential only (black dotted curve), (ii) the static potential (red dash-dotted curve), (ii) the singlet state (orange dashed curve), and (iv) the triplet state (green solid curve). From the results, we see that the confinement potential (or frequency $\omega_{x}$ ) of the QD strongly affects the transmission probability through the QD. Furthermore, the Coulomb potential alters considerably the scattering. The electron-electron exchange potential splits the resonant peak into two due to different spin states of the system. It shows that, when the incident electron has anti-parallel (parallel) spin with the confined electron in the QD, the transmission probability is enhanced (suppressed) significantly at low energy. This is a kind of spin filter effect if we could control the spin state of the confined electron.

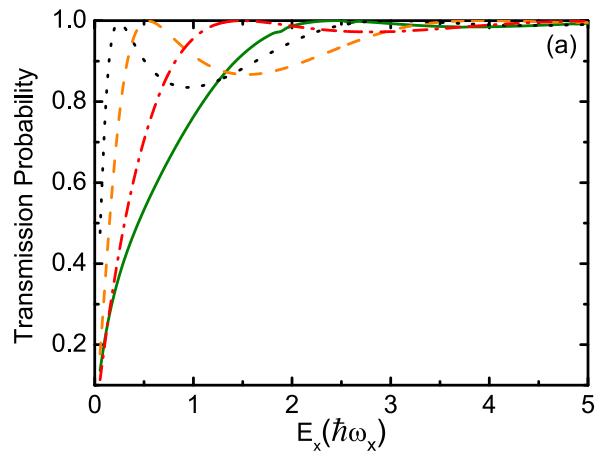

(a) $\hbar \omega_{x}=3 \mathrm{meV}$

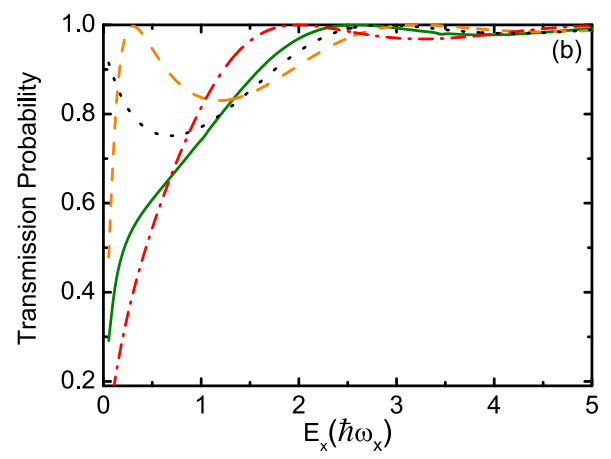

(b) $\hbar \omega_{x}=5 \mathrm{meV}$.

Fig. 11. Transmission probability as a function of the incident electron's energy assuming different scattering situations: only the QD potential (black dotted curve), the static potential (red dash-dotted curve), the singlet state (orange dashed curve), and the triplet state (green solid curve). 


\section{Conclusion remarks}

We presented a theoretical approach to calculate the electron scattering and transport through an N-electron QD embedded in a 2D and a Q1D semiconductor structure. The multichannel L-S equations are solved numerically using the iterative method of continued fractions considering the electron-electron interactions. From this method, we can study the multichannel scattering including the excited states of the QD. The electron transport property due to elastic and inelastic scattering, as well as its dependence on the spin states of the system can be obtained in great precision.

We applied this method to the case where only one electron is confined in the QD. The results indicate a rapid convergency of the numerical method for the electron scattering in both 2D and $1 \mathrm{D}$ systems. We found that the electron-electron exchange effects are relevant when the kinetic energy of incident electron is small. For a QD of more electrons, we need firstly to find the eigenstates of the QD with electron-electron interactions. In principal, the scattering processes can be calculated according to the total wave-function defined by Eq. (6).

\section{Acknowledgments}

This work was supported by FAPESP and CNPq. (Brazil).

\section{References}

Adhikari, S. K. (1986). Am. J. Phys. 54: 362.

Bransden, B. H. \& McDowell, M. R. C. (1977). Phys. Rep. 30: 207.

Burkard, G., Engel, H. A. \& Loss, D. (2000). Fortschr. Phys. 48: 965.

Buttiker, M., Imry, Y., Landauer, R. \& Pinhas, S. (1985). Phys. Rev. B 31: 6207.

Castelano, L. K. (2006). PhD thesis, University of Sao Paulo.

Castelano, L. K., Hai, G.-Q. \& Lee, M.-T. (2007a). Phys. Rev. B 76: 165306.

Castelano, L. K., Hai, G.-Q. \& Lee, M.-T. (2007b). Phys. Stat. Sol. (c) 4: 466.

da Paixão, F. J., Lima, M. \& McKoy, V. (1996). Phys. Rev. A 53: 1400.

Darwin, C. (1930). Proc. Cambridge Philos. Soc. 27: 86.

Engel, H.-A. \& Loss, D. (2002). Phys. Rev. B 65: 2002.

Fisher, D. S. \& Lee, P. A. (1981). Phys. Rev. B 23: 6851.

Fock, V. (1928). Z. Phys. 47: 446.

Fransson, J., Holmstrom, E., Eriksson, O. \& Sandalov, I. (2003). Phys. Rev. B 67: 205310.

Gundogdu, K., Hall, K. C., Boggess, T. F., Deppe, D. G. \& Shchekin, O. B. (2004). Appl. Phys. Lett. 84: 2793.

Hegemann, T., Oberste-Vorth, M., Vogts, R. \& Hanne, G. F. (1991). Phys. Rev. Lett. 66: 2968.

Horacek, J. \& Sasakawa, T. (1984). Phys. Rev. A 28: 2151.

Imry, Y. \& Landauer, R. (1999). Rev. Mod. Phys. 71: s306.

Joachain, C. J. (1975). Quantum collision theory, North-Holland, Amsterdam.

Konig, J. \& Martinek, J. (2003). Phys. Rev. Lett. 90: 166602.

Koppens, F. H. L., Buizert, C., Tielrooij, K. J., Vink, I. T., Nowack, K. C., Meunier, T., Kowenhoven, L. P. \& Vandersypen, L. M. K. (2006). Nature 442: 766.

Landauer, R. (1957). IBM J. Res. 1: 223.

Landauer, R. (1970). Phil. Mag. 21: 863.

Landauer, R. (1975). Z. Phys. B24: 247.

Merzbacher, E. (1970). Quantum Mechanics, John Wiley and Sons, Inc., New York. 
Morse, P. M. \& Feshbach, H. (1953). Methods of Theoretical Physics, McGraw-Hill, New York. Qu, F. Y. \& Vasilopoulos, P. (2006). Phys. Rev. B 74: 245308.

Sarma, S. D., Fabian, J., Hu, X., \& Zutic, I. (2001). Solid State Commun. 119: 207.

Seneor, P., Bernand-Mantel, A. \& Petroff, F. (2007). J. Phys.: Condens. Matter 19: 165222.

Szabo, A. \& Ostlund, N. (1982). Modern Quantum Chemistry, Macmillan Publishing, New York. Vargiamidis, V., Valassiades, O. \& Kyriakos, D. S. (2003). Phys. Stat. Sol. (b) 236: 597.

Wolf, S. A., Awschalom, D. D., Buhrman, R. A., Daughton, J. M., von Molnár, S., Roukes, M. L., Chtchelkanova, A. Y. \& Treger, D. M. (2001). Science 294: 1488.

Zhang, P., Xue1, Q.-K., Wang, Y. \& Xie, X. C. (2002). Phys. Rev. Lett. 89: 286803. 


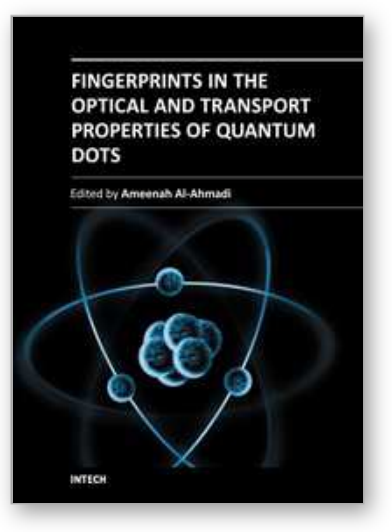

\author{
Fingerprints in the Optical and Transport Properties of Quantum \\ Dots \\ Edited by Dr. Ameenah Al-Ahmadi
}

ISBN 978-953-51-0648-7

Hard cover, 468 pages

Publisher InTech

Published online 13, June, 2012

Published in print edition June, 2012

The book "Fingerprints in the optical and transport properties of quantum dots" provides novel and efficient methods for the calculation and investigating of the optical and transport properties of quantum dot systems. This book is divided into two sections. In section 1 includes ten chapters where novel optical properties are discussed. In section 2 involve eight chapters that investigate and model the most important effects of transport and electronics properties of quantum dot systems This is a collaborative book sharing and providing fundamental research such as the one conducted in Physics, Chemistry, Material Science, with a base text that could serve as a reference in research by presenting up-to-date research work on the field of quantum dot systems.

\title{
How to reference
}

In order to correctly reference this scholarly work, feel free to copy and paste the following:

Leonardo Kleber Castelano, Guo-Qiang Hai and Mu-Tao Lee (2012). Electron Scattering Through a Quantum Dot, Fingerprints in the Optical and Transport Properties of Quantum Dots, Dr. Ameenah Al-Ahmadi (Ed.), ISBN: 978-953-51-0648-7, InTech, Available from: http://www.intechopen.com/books/fingerprints-in-theoptical-and-transport-properties-of-quantum-dots/electron-scattering-through-a-quantum-dot

\section{INTECH}

open science | open minds

\section{InTech Europe}

University Campus STeP Ri

Slavka Krautzeka 83/A

51000 Rijeka, Croatia

Phone: +385 (51) 770447

Fax: +385 (51) 686166

www.intechopen.com

\section{InTech China}

Unit 405, Office Block, Hotel Equatorial Shanghai

No.65, Yan An Road (West), Shanghai, 200040, China 中国上海市延安西路65号上海国际贵都大饭店办公楼 405 单元

Phone: +86-21-62489820

Fax: +86-21-62489821 
(C) 2012 The Author(s). Licensee IntechOpen. This is an open access article distributed under the terms of the Creative Commons Attribution 3.0 License, which permits unrestricted use, distribution, and reproduction in any medium, provided the original work is properly cited. 